\title{
Consequences of Stigma in the Life of People with Mental Disorders: A Qualitative Research
}

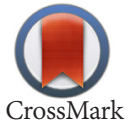

\author{
Alireza Momeni ${ }^{1}$, Soroor Parvizy ${ }^{2 *}$ \\ 1. Razi Educational Treatment Psychotherapy Center, University of Social Welfare and Rehabilitation Sciences, Tehran, Iran. \\ 2. Department of Nursing, School of Nursing and Midwifery, Iran University of Medical Sciences, Tehran, Iran.
}

Citation: Momeni, AR \& Parvizy, S 2016, 'Consequences of stigma in the life of people with mental disorders: A qualitative research', Journal of Client-Centered Nursing Care, vol. 1, no. 4, pp. 189-196.

ol: $:$ http://dx.doi.org/10.15412/J.JCCNC.04010404

Article info:

Received: 16 May. 2015

Accepted: 12 Sep. 2015
Keywords:

Qualitative research, Social stigma, Person with mental disorder

\begin{abstract}
A B S T RA C T
Background: The stigma of mental disorder has destructive impacts on emotions, feelings, personal relationships, parenting, education, occupation, and house management of people with mental disorders. Understanding and awareness of the stigma consequences are important for the establishment and development of constructive relationships with people suffering from mental disorders as well as their care and treatment. This qualitative research aimed to explain the attitudes of people with mental disorder, the treatment team, and the patients' families towards the consequences of stigma in the life of people with mental disorder.
\end{abstract}

Methods: This study was conducted using a qualitative approach and content analysis method. Sixteen people (patients with mental disorder, their family members, psychiatrist, psychiatric nurse, social worker, legal expert) were selected from 3 psychiatric centers in Tehran by using targeted sampling method and were interviewed using semi-structured interviews until the data were saturated.

Results: Content analysis of the interviews showed that people with mental disorder experience the consequences of stigma as fear of stigma, self-blame, searching for a cause for the disorder, denial, avoiding psychiatric treatment, rejection, discrimination, and relapse.

Conclusion: The findings of this research can help the psychiatric team to make effective and targeted treatment decisions. Performing interventions to reduce stigma of mental disorder in society seems to be necessary.

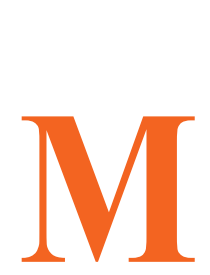

\section{Background}

ental disorders affect a large number of people. Among 10 major causes of disability, 5 are related to psychiatric disorders (World Health Organization 2001) that are considerably prevalent.
Mental disorders affect approximately $30 \%$ of Americans annually (Hertling \& Kessler 2006; Riddell \& Haddian 2000). One out of 5 Americans suffers from some kind of mental disorders (World Health Organization 2002). In Iran, the prevalence of mental disorders is estimated to be $17.1 \%$ and is higher among middle-aged people (compared to other age groups), divorcees (compared to

\section{* Corresponding Author:}


married and single people), urban residents, the illiterate, housewives, and the unemployed (Noorbala et al. 2011).

Stigma is a universal and complex phenomenon (Larsen \& Lubkin 2009) that affects the psychosocial aspects of patient's life (Dwivedi 2008). Stigma is also related to social background and expresses in relationships. Therefore, stigma is considered a social construct through which society labels the patients and their families (Major \& O'Brien 2005). This social construct distinguishes the individual and places him or her in a lower group than others (Pinfold et al. 2003). Although stigma has a wide range of aspects, its psychological consequences are more severe and difficult to deal with (Nguma 2010).

Stigma and its consequences for people with mental disorder create a special condition for them and can be considered a major concern of those with mental disorders (Dwivedi 2008; Major \& O'Brien 2005; Pinfold et al. 2003; Nguma 2010). Thus, in people with mental disorder, stigma is a common and widespread phenomenon (Mak et al. 2007; Smith 2002; Crisp et al. 2000; Corrigan, Kerr \& Knudsen 2005) that prevents people in society from having professional cooperation with people with mental disorders. They are even reluctant to rent their houses to these people or live together with them (Rusch, Angermeyer \& Corrigan 2005; Corrigan et al. 2003).

Psychiatric nurses are members of the psychiatric team and play a significant role in giving psychological support to patients (Rusch, Angermeyer \& Corrigan 2005). In fact, they not only take care of physical needs of the patients, but also help patients considerably with their confusions and psychological disorders. Psychiatric nursing stresses the establishment and development of "relationship therapy" (Corrigan et al. 2003). Relationship therapy is, in fact, a relationship between the patient and the nurse with the purpose of gaining information, understanding the patient's problems, and taking therapeutic measures. Furthermore, its main objective is to provide comfort and promote patients' feelings such as trust, confidence, and safety (Feldman \& Crandall 2007).

Participants in this study considered the main cause of the "relapse" as discarding the psychiatric treatment due to the fear of stigma. In line with these findings, (Corrigan, Kerr \& Knudsen 2005) mentioned 2 factors that decrease the effectiveness of psychiatric treatment in patients and consequently, relapse of the symptoms. First, refusing to follow up on the psychiatric treatment due to the fear of stigma and second, the "why try effect", which occurs when patients accept and internalize cliché beliefs and prejudices of society about mental patients. Therefore, they disregard themselves as competent and efficient individuals and do not try to treat themselves.

In general, the findings of this study showed that while facing stigma, people with mental disorders experience mental struggles and emotional reactions such as fear, anxiety, low self-confidence, and the feeling of rejection, which direct their individual and social behaviors. These conditions can affect the process of treatment and care. It is important for the members of the psychiatric team, including psychiatric nurses, to understand these conditions and issues and to make effective and targeted therapeutic decisions based on the patients' needs. People with mental disorders face 2 problems; first, the direct effects of cognitive, emotional, and behavioral problems, which restrict the abilities and effective performance of the patient and second, social rejection, intrapersonal dissociation, and identity fracture, which are caused by the stigma of mental disorder (Feldman \& Crandall 2007).

Stigma is a set of negative attitudes, beliefs, thought, and behaviors that lead to fear, rejecting others' favors, avoidance, and prejudice. These are serious problems and obstacles for patients with mental disorders to achieve life goals (Pinto-Foltz \& Logston 2009). Patients with mental disorders experience 3 kinds of stigma; 1) emotional stigma or self-stigma, that is, the patient feels inadequate and defective; 2) stigma by family members who reject the patient; and 3) stigma by society which ends in discrimination between apparently healthy individuals and mental patients who are not fully accepted by the society.

Stigmatizing reduces self-confidence and leads to embarrassment and refusing to ask others for help and leads to social isolation (Shahveyis et al. 2007). In their social activities, people experiences discrimination due to stigma of mental disorder (Ahmadvand et al. 2010). Therefore, to avoid rejection and discrimination, these people may limit their social relationships to individuals who are either stigmatized like themselves or are aware of their being stigmatized and have accepted the conditions of their disorders (Tavakoli et al. 2006).

The pattern of stigma and discrimination differs in various countries depending on the culture, beliefs about mental disorders, and conceptualization (Knifton et al. 2010). In 2012, a research was conducted in Netherlands by Switaj et al. to study the frequency, types, and sources of stigma and discrimination in 422 patients with mental disorders. The results showed that individuals experience stigma on a daily basis and frequently at the workplace and in their interpersonal relationships. They 
considered their colleagues, families, and society as the source of stigma (Switaj et al. 2012).

Lundberg et al. (2007) conducted a research on 200 mental patients to study the prevalence of rejection, discrimination, and humiliation. The results showed that people with mental disorder who had a better performance in society, had experienced lower levels of discrimination and humiliation and there was a relationship between the experience of rejection and performance, number of hospitalizations and contacting social services agencies (Lundberg et al. 2007).

In Iran, Sadeghi et al. (2003), in a cross-sectional and comparative study, compared the effects of stigma of mental disorder among 3 groups of families of schizophrenic, bipolar, and depressed patients. The data were collected using 34-item self-administered questionnaires. The findings of this research indicated that a considerable percentage of families of the patients participating in the study had been humiliated or discriminated as a result of stigma and also a feeling of shame due to reported mental disorder by most families (Sadeghi, Kaviani \& Rezai 2003). In a similar study, Shahveisi et al. (2007) examined the effects of stigma of mental disorder in 2 groups of families of patients with schizophrenia and major depressive disorder without psychotic features and obtained similar results (Shahveyis et al. 2007).

Because of sociocultural differences between Iran and other countries and few studies conducted on the stigma of mental disorder, qualitative studies are required to explain the profound concepts in this field. Using qualitative research is a step towards meeting the need for interdisciplinary research on mental disorders. These studies help understand human phenomena with an emphasis on the social context (Parvizy, Ahmadi \& Pourasadi 2011). In Iran, no qualitative research has ever conducted on the consequences of stigma in the life of people with mental disorder. Thus, this research sought to explain the attitudes of people with mental disorder, the treatment team, and the patients' families towards the consequences of stigma in the life of people with mental disorder.

\section{Materials \& Methods}

This study was a qualitative content analysis conducted for 11 months. Participants were selected from 3 active psychiatric centers in Tehran, Iran, namely, Razi Educational Treatment Psychotherapy Center, Sina Consultation and Psychological Rehabilitation Center, and Aftab Society. The participants included 8 patients with mental disorder diagnosed by 2 psychiatrists and aware of their disorder, 5 members of the treatment team (psychiatrist, psychiatric nurse, nurse, social worker, and an occupational therapist), a legal expert, and 2 family members and relatives of the patients who had sufficient experience with the stigma of mental disorder and volunteered to participate in the interviews.

Sampling method was purposive sampling and the data collection tool was semi-structured interview. Prior to each interview, the participants studied the written consent forms and then signed them. The questionnaire related to personal information of the participants (such as age, sex, occupation, and education) was separately completed. Then, general and open questions were asked and this process continued until the data were saturated. Participants were asked guiding questions for starting the conversation such as: "What comes to your mind first when you think of mental disorders?" or "How much does society care for people with mental disorder?" The verbal communications of the participants were recorded on tape and transcripted at the first opportunity along with nonverbal communications and were simultaneously codified and analyzed as much as possible.

To make the data acceptable and objective, the following methods were used: constant study and observation, spending sufficient time, good communications, using combined research methods (spatial and temporal methods), revisions by supervisors, and review of the manuscripts by participants.

Moral considerations were observed by obtaining written consent from participants as well as taking permission for recording their voice, ensuring confidentiality of their information, their right to withdraw from the research at any time, acquiring introduction letters, and following necessary legal procedures.

Analysis of qualitative content is one of the qualitative research methods. A method which, for over half a century, has simply maintained a combination of coding and qualitative research and has been able to bridge the gap between qualitative and quantitative research. Qualitative content analysis is a suitable method for investigating human phenomena and clarifying the meaning behind them. First, to understand the real meaning behind the interview manuscripts, the transcripts were studied carefully. Then, the collected data were analyzed into meaningful units or related statements and were compressed as much as possible. This process gradually became more and more abstract and continued until the main themes emerged (Granehim \& Lundman 2004).

\section{Results}

Participants had the following demographic characteristics (Table 1). The following codes were obtained from 
Table 1. Demographic characteristics of participants.

\begin{tabular}{|c|c|c|c|}
\hline Variable & Characteristics & No. & $\%$ \\
\hline \multirow{2}{*}{ Sex } & Male & 11 & 68.75 \\
& Female & 5 & 31.25 \\
\hline \multirow{3}{*}{ Age, y } & $22-37$ & 7 & 43.75 \\
& $38-53$ & 8 & 50 \\
\hline \multirow{2}{*}{ Marital status } & $54-69$ & 1 & 6.25 \\
& Married & 7 & 43.75 \\
\hline \multirow{3}{*}{ Educational degree } & Single & 9 & 56.25 \\
\hline & Specialist & 1 & 6.25 \\
& MS & 2 & 25 \\
& BS & 1 & 6.25 \\
& Student & 25 \\
& Diploma & 4 & 25 \\
\hline \multirow{2}{*}{ Occupation } & Below diploma & 1 & 6.25 \\
& Psychiatrist & 1 & 6.25 \\
& Psychiatric nurse & 1 & 6.25 \\
& Nurse & 1 & 6.25 \\
& Occupational therapist & 1 & 6.25 \\
\hline
\end{tabular}

analysis of the participants' opinions regarding the consequences of stigma in the life of the patients:

\section{Fear of stigma}

\section{Self-blame}

3. Searching for a cause of the disorder

\section{Denial}

\section{Avoiding psychiatric treatment}

6. Rejection and discrimination against the patient

7. Discriminatory treatment of the patient

8. Relapse of the disorder

\section{Fear of stigma}

According to most participants, patients with mental disorders are afraid of speaking about their disorder to others. According to a patient, fear is a feeling that a patient has about society's change of attitude towards a mental patient. “... I fear people may change their behavior when I tell them that I am sick, that I am a mental patient... or if they find out that I have been hospitalized in a psychiatric hospital" (codes 641, 634, 633, 59 and 652).

\section{Searching for a cause of the disorder}

According to the participants, sometimes people with mental disorders who experience stigma, try to find a cause for their disorder and ascribe their disorder to uncontrollable external factors that cause less stigma. In this regard, a legal expert stated: "[the patient] believes that his behavior is influenced by the behavior of the people around him. He even ascribes his behavior to social harms. He says that social pressures have put me in this situation..., My unemployment made me sick... . Sometimes he blames his wife; he says my wife caused this. She put so much pressure on me that made me sick..., My brother caused this..., My colleagues made me sick..., I was healthy" (code 777).

Some other causes were "the will of God", "genetic factors", "romantic failure", and "fate and predestination" (codes 446, 301, 777, 54, and 554).

\section{Denial}

Most of the participants believed that hiding the disorder and history of hospitalization is one of the strategies that patients choose while facing stigma. “... I hide my disorder.... For example, I fear people might change their behavior when I tell them that I am sick, that I am a mental patient..., or if they find out that I have been 
hospitalized in a mental hospital... they wonder why I have been in a mental hospital for 3 years and conclude that my disorder is chronic and that they cannot work with me. It is like an automatic signal that warns them to end their relationship with me", said one of the mental patients (codes 620,630, and 631).

“... Because of shame, all mental patients avoid other people so that no one finds out that they take medication for their mental disorder...", said a person who had been a mental patient for 15 years (code 805$)$.

Participants also spoke of the stigma of "hospitalization". Therefore, they mentioned "hiding the fact that they have been hospitalized" as a way to avoid stigma, which is used by the patient and his or her relatives. A person with mental disorder spoke of hiding his disorder to find a low job: “... I will go to a service company to get a job. I think I can do those kind of jobs... I will say I have no history of mental disorder or hospitalization..." (code 806).

\section{Avoiding psychiatric treatment}

According to most participants, visiting a psychiatric center for treatment is itself considered being stigmatized. Therefore, to avoid the stigma of mental disorder, both patients and their families avoid visiting psychiatrists or psychologists.

"When you offer families to give them consultation or if you say that it is okay for them to visit a psychiatrist, most of them get upset and may take it personally. It is mostly due to the stigma of mental disorder, because they fear they might be labeled", stated a social worker (code 247).

A psychiatric nurse believed that this is due to stigma. "Stigma makes people avoid treatment. they fear the medication that is prescribed. They think to themselves that if they take the medication, people will think that I am a mental patient or they say to themselves or they think they are not mentally sick to need to take medication", she said (codes 299, 294, and 300).

\section{Rejection and discrimination against the person with mental disorder}

Most of the participants believed that "rejection" from society and sometimes even family is one of the consequences of the stigma of mental disorder. Father of a mental patient had a similar opinion. "Our society considers mental disorder as an abnormal phenomenon. Our culture had not accepted mental disorder yet", he said (code 567).

Fear of rejection has led to hiding the patient from friends and relatives, reducing face-to-face or telephone contacts with the patient, ignoring the patient and refusing to pay a visit to him at the hospital or the patient not being released from hospital.

"Rejection leads to isolation; Isolation leads to losing social status, which itself leads to decreased financial security. These are interconnected links", a nurse said (code 408).

Another person stated: "No one gets close to these patients. People do not communicate with them. They are not aware of their positive characteristics" (code 682).

Discrimination (by family and society) against the person with mental disorder was frequently emphasized by the participants. Discrimination is observed in the form of lack of access to proper jobs, unsuccessful marriages, lack of good relationships with people, and deprivation from the basic rights. A social worker spoke of violation of mental patients' rights in society: "I say these patients need more support. Can you believe that Mental Health Act has not been approved yet?" (codes 188-189).

A legal expert explained that, sometimes, when a law enforcement agency decrees that a patient should be under quarantine, the patient experiences stigma and is rejected by society. "A soon as he is put under quarantine and loses his guardianship, he is rejected by others who want to get rid of him. As they say, they isolate him. He is rejected by his family. No one marries his daughters or sons. No one travels with him or visits him. People reject him because they do not want to be disturbed. This is a violation of mental patients' rights", he said (code 767).

\section{Relapse of the disorder}

The stigma of mental disorder prevents the individual from seeking and pursuing treatment. This situation leads to further social isolation. In this regard, a social worker stated: “... when they are isolated, they get cut off from the world, like a deserted island, they mind their own business... and this is a destructive factor which impedes treatment. When a person becomes more isolated, he loses his contact with others, the symptoms relapse and treatment is prolonged" (code 175). A person with mental disorder also considered lack of acceptance of a mental patient in society as a factor which deteriorates his condition. "A mental patient is not accepted by society and this deteriorates his condition," he said (code 812).

\section{Discussion}

Based on the findings of the present research, stigma leads to negative effects, consequences, and unpleasant 
experiences and sheds a dark shadow over the life of the people with mental disorders. Various studies indicate that different mental disorders lead to their specific stigma such as being dangerous, incurability, being blamed, and social distance (Knifton et al. 2010). Also, there is an association between negative symptoms of the disorder and its distance from society. These weak social skills and negative symptoms of the disorder cause stigma (Corrigan et al. 2003).

Based on the findings of this study, people with mental disorders are even stigmatized and rejected by their close relatives. The stigma of mental disorder can result in family instability, social rejection, and discrimination (Feldman \& Crandall 2007). This condition is rooted in the culture of the society and negative attitudes towards mental patients.

Knifton believed that the pattern of stigma and discrimination is different in various countries depending on the culture, beliefs about mental disorders conceptualization (Knifton et al. 2010). Furthermore, cultural characteristics have a close association with mental disorders, seeking treatment, incidence of the disorders, their symptoms, and stigma (Tavakoli et al. 2006).

Patients with mental disorder are faced with 2 challenges. On the one hand, they have to deal with the conditions and disabilities caused by the disorder. On the other hand, the challenges of stigma are caused by misunderstandings about the disorders, which affects mental patients that determine the quality of their lives such as job opportunities (Corrigan et al. 2003). In this regard, delivering educational programs provided by the media with the aim of proper understanding of mental disorders can play a significant role in building culture and preventing mental patients' rejection.

People with mental disorders in this study spoke of discriminatory treatment of family and society. Studies show that most mental patients experience discriminatory behaviors and more than half of the people with severe mental disorders have reported the experience of discriminatory behaviors (Tavakoli et al. 2006). As a result of discrimination, which originates from misunderstanding of the patients, people with mental disorder experience further social isolation, which is itself the cause of other problems such as unemployment, lack of access to social facilities, healthcare and insurance problems (Sadeghy, Kaviani \& Rezai 2003).

As a result of stigma, these patients lose their jobs and chances of marriage, and are even deprived of their most basic rights in life. McSwine believed that the reason for this discrimination is the stigma of mental disorder, which is observed in social rules, medical insurance and policies related to mental patients, including allocation of funds to mental health issues (Shahveyis et al. 2007). However, according to the declaration of the World Federation of Mental Health, all mental patients must be treated like other patients and based on the same ethical and professional principles (Hojatiabed et al. 2010).

It seems that civil law is in favor of discrimination against these patients. By revising the law and observing ethical principles and religious teachings, which emphasize dignifying human beings and with the help of specialists in the field of psychiatry as well as approval of the Mental Health Act by the Islamic Consultative Assembly, major steps can be taken to preserve the dignity of people with mental disorder.

Participants in this study consider the main reason for the relapse of the disorder is avoiding psychiatric treatment due to fear of stigma. Pinto-Foltz and Logston (2009) reported that the use of mental health services and treatment of mental disorders was largely associated with stigma (Riddell \& Haddian 2000). In line with these findings, Corrigan, Kerr and Knudsen (2005) believed that 2 factors reduced the effects of psychiatric treatment and consequently, led to lack of improvement of the patient. First, avoiding psychiatric treatment due to fear of stigma and second, the "why try effect", which occurs when patients accept and internalize cliché beliefs and prejudices of society about mental patients, and thus, disregard themselves as competent and efficient individuals and do not try to treat themselves (Tavakoli et al. 2006).

Thus, negative consequences of lack of diagnosis or treatment and management of the mental disorder lead to the relapse of the disorder, family instability, lack of work efficiency, and negative social effects (Hertling \& Kessler 2006). In all the groups that were examined in terms of stigma, it was found that stigma has harmful effects on the mental patients; for instance, negative attitudes among personnel and psychiatrists towards mental patients decreases the use of healthcare services, which intensifies the symptoms of the disorder (Knifton et al. 2010). Thus, the relationships between the stigma of mental disorder and its effects on the personal and social life of the patients as well as the symptoms of the disorder can be imagined as the interconnected links in a chain, where any change in one link affects the whole chain.

One of the limitations of this research was obtaining non-homogeneous samples (in terms of age, sex, social status, etc.) in accordance with the inclusion criteria of 
the research. Finding people who both have the experience of mental disorder and be willing to share their experience was difficult and time-consuming.

In conclusion, stigma in people with mental disorder has serious consequences which affect various aspects of their personal and family life. The damages and consequences of stigma are sometimes serious and irreparable. Education, social awareness and individual, family and social support for the patients and their families as well as public education can help reduce the stigma and its negative effects.

Functional research can be conducted to develop and implement strategies for removing the stigma of mental disorder in society. Also, identifying the effects of public interventions, education through media, involvement of family members and establishing self-help groups as well as support groups for identifying the problems caused by stigma and reducing stigma and its consequences seem to be helpful.

\section{Conflict of Interests}

The authors declared no conflict of interests.

\section{Acknowledgements}

We appreciate the sincere cooperation of all people that this research could not have been conducted without their assistance. We ask God Almighty to grant them all health and happiness.

\section{References}

Ahmadvand, A, Sepehrmanesh, Z, Sadat Ghoreyshi, F, Assarian, F, Abbas Moosavi, G \& Etesam, F 2010, '[Prevalence of Mental Disorders in General Population of Kashan City (Persian)]', Iranian Journal of Epidemiology, vol. 6, no. 2, pp. 16-24.

Corrigan, PW, Bodenhausen, G, Markowitz, F, Newman, L, Rasinski, K \& Watson, AC 2003, 'Demonstrating translational research for mental health services: An example from stigma research', Mental Health Services Research, vol. 5, no. 2, pp. 79-88.

Corrigan, PW, Kerr, A, \& Knudsen, L 2005, 'The stigma of mental illness: Explanatory models and methods for change', Applied and Preventive Psychology, vol. 11, no. 3, pp. 179-90.

Crisp, AH, Gelder, MG, Rix, S, Meltzer, HI, \& Rowlands, OJ 2000, 'Stigmatisation of people with mental illness', British Journal of Psychiatry, vol. 177, no. 1, pp. 4-7.

Dwivedi, A 2008, Living on the outside: The impact of diabetes-related stigma, http://banderasnews.com/0811/hb-wdd14dec01. htm.
Feldman, D \& Crandall, C 2007, ‘Dimensions of Mental illness Stigma:What about Mental illness Causes Social Rejection?', Journal of Clinical and Social Psychology, vol. 26, no. 2, pp. 137138

Granehim, UH \& Lundman, B 2004, 'Qualitative content analysis in Nursing Research: concepts, procedures and measures to achieve trustworthiness', Nursing Education Today, vol. 24, no. 2 , pp. 105-112

Hertling, D \& Kessler, RM 2006, Management of common musculoskeletal disorders: Physical Therapy Principles and methods, Lippincott Williams \& Wilkins, Philadelphia.

Hojatiabed, E, Karbalaei Nouri, A, Rafiei, H \& Karimilou, M 2010, '[The Efficacy of Psychosocial Occupational Therapy Services on Quality of Life oh Chronic Psychiatric Patients. (Persian)]', Journal of Rehabilitation, vol. 11, no.1, pp. 23-28.

Knifton, L, Gervais, M, Newbigging, K, Mirza, N, Quinn, N, Wilson, $\mathrm{N}$ et al 2010, 'Community conversation: Addressing mental health stigma with ethnic minority communities', Social Psychiatry and Psychiatric Epidemiology, vol. 45, no. 4, pp. 497-504

Larsen, PD \& Lubkin, IM 2009, Chronic illness: Impact and interventions, Jones \& Bartlett Learning, Burlington.

Lundberg, B, Hansson, L, Wentz, E \& Bjorkman, T 2007, 'Sociodemographic and clinical factors related to devaluation/discrimination and rejection experiences among users of mental health services', Social Psychiatry and Psychiatric Epidemiology, vol. 42, no. 4, pp. 295-300.

Major, B \& O’Brien, LT 2005, 'The social psychology of stigma', Annual Review of Psychology, vol. 56, pp. 393-421.

Mak, WWS, Poon, CYM, Pun, LYK \& Cheung, SF 2007, 'Metaanalysis of stigma and mental health', Social Science $\mathcal{E}$ Medicine, vol. 65 , no. 2 , pp. 245-261.

Nguma, LK 2010, 'Health seeking and health related behaviour for type 2 diabetes mellitus among adults in an urban community in Tanzania' PhD thesis, University of Otago, http:// hdl.handle.net/10523/456.

Noorbala, AA, Bagheri Yazdi, A, Asadi Lari, M \& Vaez Mahdavi, MR 2011, '[Mental Health Status of Individuals Fifteen Years and Older in Tehran-Iran, 2009 (Persian)]', Iranian Journal of Psychiatry and Clinical Psychology, vol. 16, no. 4, pp. 480.

Parvizy, S, Ahmadi, F \& Pourasadi, H 2011, '[A Qualitative Study on Social Predisposing Factors of Adolescents Health (Persian)]', Iranian Journal of Nursing, vol. 24, no. 69, pp. 8-17.

Pinfold, V, Toulmin, H, Thornicroft, G, Huxley, P, Farmer, P \& Graham, T 2003, 'Reducing psychiatric stigma and discrimination: evaluation of educational intervention in UK secondary schools', British Journal of Psychiatry, vol. 182, no. 4, pp. 342-346.

Pinto-Foltz, M \& Logston, C 2009, 'Reducing Stigma to Mental Disorders: Initiatives, Interventions, and Recommendations for Nursing', Archives of Psychiatric Nursing, vol. 23, no. 1, pp. 32-40.

Riddell, JS \& Haddian, M 2000, 'Field potentials generated by group Ia muscle afferents in the lower-lumbar segments of the feline spinal cord', Journal of Physiology, vol. 522, no. 1, pp. 97108 
Rusch, N, Angermeyer, MC \& Corrigan, PW 2005, 'Mental illness stigma: Consequnses, and initiatives to reduce stigma', European Psychiatry, vol. 20, no. 8, pp. 529-39.

Sadeghi, M, Kaviani, K \& Rezai, R 2003, '[Stigma of Mental Disorders among Families of Patients with Major Depressive Disorder, Bipolar Disorder and Schizophrenia (Persian)]', Advances in Cognitive Science, vol. 5, no. 2, pp. 16-25.

Shahveyis, B, Shoja Shaftie, S, Fadai, F \& Dolatshahi, B 2007, '[Comparison of Mental Illness Stigmatization in Families of Schizophrenic and Major Depressive Disorder Patients without Psychotic Features (Persian)]', Journal of Rehabilitation, vol. 8, no. 29, pp. 21-27.

Smith, M 2002, 'Stigma', Advances in Psychiatric Treatment, vol. 8 , no. 8, pp. 317-325.

Switaj, P, Wciórka, J, Grygiel, P, Anczewska, M, Schaeffer, E, Tyczynski, $\mathrm{K}$ et al 2012, 'Experiences of stigma and discrimination among users of mental health services in Poland' Transcultural Psychiatry, vol. 49, no. 1, pp. 51-68.

Tavakoli, S, Kaviani, H, Sharifi, V, Sadeghi, M \& Fotouhi, A 2006, '[Examining Cocnitive, Emotional and Behavioral Components of Public Stigma towards Persons whit Mental illness (Persian)]', Advances in Cognitive Science, vol. 8, no. 2, pp. 31-43.

World Health Organization 2001, Mental health around the World: Step exclusion, dare to care, world health day, World Health Organization, Geneva.

World Health Organization 2002, World Health report 2002: Reducing the risks, promoting healthy life, World Health Organization, Geneva. 